Alexia Stumpf

HEP-BEJUNE (Suisse)

François Joliat

HEP-BEJUNE (Suisse)

Denis Perrin

HEP-BEJUNE (Suisse)

\section{Comment analyser les traces de la professionnalisation dans les écrits des institutions de formation à l'enseignement?}

\section{ésumé}

Le présent article s'ancre dans une recherche sur la professionnalisation de la

formation à l'enseignement en Suisse. Elle visait à en cerner les principales conceptions, notamment à travers l'analyse de la rhétorique de professionnalisation

contenue dans les écrits institutionnels. Notre contribution vise à présenter et à soumettre au débat un outil méthodologique pour organiser ce corpus de textes de manière à rendre visibles les traces de la professionnalisation et ses retraductions. Cet outil permet, notamment, de ventiler les textes en fonction de leur(s) auteur(s), de leur adressage et de leurs finalités pour garantir la pertinence de leur analyse et de leur interprétation.

À titre d'exemple, la mise en application de notre outil dans l'examen des textes sur la place de la recherche dans la formation montre quill existe des différences entre les finalités déclarées à un niveau textuel et celles explorées à d'autres niveaux, sources de possibles tensions qui invitent à la précaution dans l'analyse.

Nous faisons l'hypothèse que cet outil est généralisable à d'autres institutions de formation des enseignants puisqu'il permet d'organiser les textes institutionnels pour les préparer à l'analyse.

Cet outil a fonctionné comme ressource pour l'ensemble du groupe de chercheurs dans la détermination de leurs objets de recherche et comme garant de la cohérence d'ensemble des différentes recherches conduites.

Mots-clés

Professionnalisation, formation des enseignants, enseignement supérieur, analyse discursive, outil conceptuel, typologie textuelle

Abstract

This article takes its origin in a research about professionalization of teacher training for higher education in Switzerland which aimed to identify the main conceptions of professionalization throughout a rhetorical analysis of the papers written by the institution. Our paper aims to present and discuss a methodological tool built to organize this corpus in order to make in a second time visible the traces of professionalization and the way they are expressed. This tool is also used to organize the corpus according to their author(s), their recipient(s) and their finalitie(s) to reinforce the quality of analysis and interpretation. For example, using this tool to study the importance of the place of research in the training has showed that there are differences between the announced finalities in the institutional written and the ones which come out of other written produced at other levels. It invites the researcher to be careful in his work by noticing the possible gaps and tensions between announced intentions and their translations into reality.

Furthermore, we assume that this tool is generalizable to other institutions for teacher education because it prepares the organization of the corpus for its analysis. At last, this tool was used by the staff of researchers to refine their subject and also to allow the harmonization of the different studies conducted within the staff.

\section{Key words}

Professionalization, teacher training, higher education, speech analysis, conceptual tool, textual typology

\section{Introduction}

Dans le cadre d'une recherche sur la professionnalisation de la formation en Suisse, financée par le Fonds national suisse de la recherche scientifique (FNS), Wentzel (2012) avait pour objectif notamment de « cerner les principales conceptions ou les modèles de professionnalisation de la formation qui se dégagent des programmes de formation à l'enseignement dans différentes institutions suisses » (p.1). Lopérationnalisation de cette démarche de recherche passait par l'analyse de la rhétorique de professionnalisation ressortant des textes contenus dans les dossiers destinés à faire valoir, par la CDIP la reconnaissance des différentes formations primaire et secondaire des institutions partenaires de cette recherche. Les résultats attendus s'appuieraient sur une méthodologie d'analyse descriptive et comparative, ainsi que sur des cadres théoriques diversifiés, en fonction des approches choisies par les différents chercheurs de l'équipe (domaines des sciences de l'éducation, de la linguistique, de la sociologie). Ils devraient permettre de décrire et de comparer les conceptions ou les modèles de la professionnalisation de la formation mis en pratique dans ces institutions.

Or, en tant que partie prenante de cette recherche, nous nous sommes rapidement rendu compte que l'interprétation du sens de la rhétorique de professionnalisation contenue dans les documents à analyser devrait prendre en considération la manière dont ces institutions ont retraduit le processus de professionnalisation dans leurs structures (les domaines de formation), dans leurs programmes (le nombre d'années de formation, l'accréditation par domaine de formation) et dans leurs écrits (produits par les formateurs, les acteurs institutionnels et par les étudiants). À cette fin, et selon les théories de l'analyse du discours, il fallait aussi spécifier la structure institutionnelle et les dispositifs de formation dans lesquels s'inscrivent ces documents, leur adressage (auteurs et destinataires), leurs finalités. 
À partir du contexte et du corpus de documents à analyser, issus de l'institution à laquelle nous appartenons ${ }^{2}$, nous avons, de manière empirique, ébauché un outil méthodologique générique, au service de l'analyse de la retraduction de la professionnalisation dans les dispositifs des instituts de formation constituant le terrain de la recherche de Wentzel (2012).

Dans un premier temps, nous poserons quelques éléments contextuels et notionnels dans lesquels s'inscrit l'universitarisation de la formation des enseignants que nous mettrons en perspective avec celui de la professionnalisation. Dans un deuxième temps, nous exposerons la manière de typologiser les documents à analyser, puis nous décrirons les composantes et le fonctionnement de notre outil. Enfin, nous expliciterons, à partir de l'exemple, la manière dont cet outil peut être utilisé au service de l'analyse des traces de la professionnalisation dans ces textes.

\section{1. Éléments notionnels et contextuels}

Cette contribution s'inscrit dans le contexte de mouvance internationale des réformes institutionnelles de la formation des enseignants visant à la professionnaliser. Il nous paraît donc important de définir le concept de professionnalisation.

\subsection{Professionnalisation de la formation des enseignants}

Ce concept, sujet à débats sociaux et scientifiques, renvoie à un mouvement global qui dépasse les champs du travail. Depuis la fin des années 1990, il s'est massivement répandu dans le champ de la formation. Wittorski (2014) en distingue trois sens : "professionnalisation-efficacité du travail ", la "professionnalisation-profession " et la "professionnalisation-formation " (p. 230). C'est le sens "professionnalisation-formation » que nous mobiliserons, processus qui vise à faire des étudiants des professionnels, « via des dispositifs de formation développant des compétences » (Wittorski, 2014). La définition courante d'un dispositif renvoie à tout système de formation. Du point de vue savant, un dispositif - ou système formel d'apprentissage dans les pays anglo-saxons - est un ensemble de moyens matériels et humains qui correspondent à une forme de socialisation particulière pour faciliter un processus d'apprentissage (Blandin, 2002).

Sans dresser un état des lieux exhaustifà ce sujet, lorsque nous évoquerons la notion de professionnalisationformation, nous nous référerons à ce que Tardif et Borges (2009) appellent «les dimensions les moins controversées de la professionnalisation» (p.110) : l'allongement de la durée de formation et son universitarisation, l'intégration de la recherche dans la formation, la place centrale occupée par la formation pratique, l'acquisition de connaissances et de compétences fondant l'expertise du professionnel, la dimension réflexive des apprenants, enfin, la valorisation de la formation tout au long de la vie. Dès lors qu'il y a quasi consensus sur ces dimensions dans les textes émanant des organismes internationaux (Wentzel et Mellouki, 2010, p. 1), ce sont celles que nous mobiliserons pour établir les indicateurs des traces de la professionnalisation.

La refonte de la formation des enseignants - au début des années 2000 - nécessite que l'on s'interroge aujourd'hui sur les manières dont ces dimensions ont été intégrées en Suisse. Comment ces institutions de formation à l'enseignement se sont-elles emparées des dimensions de la professionnalisation et comment ont-elles été retraduites dans leurs structures et leurs programmes d'études? 


\subsection{Universitarisation de la formation versus tertiarisation de la formation enseignante}

En Suisse, la professionnalisation s'est traduite par la volonté de tertiariser ou d' « universitariser » les institutions de formation. Quelles réalités se cachent derrière ces deux intentions?

Dans la plupart des cas, la tertiarisation se substitue à une universitarisation généralisée. Il y a universitarisation lorsque :

les lieux de transmission des savoirs d'un secteur professionnel, ces savoirs eux-mêmes et les formateurs qui les transmettraient se trouvent en quelque sorte absorbés par l'université. [...] Les savoirs professionnels y sont désormais non seulement transmis, mais aussi créés et accumulés selon les règles particulières de l'université, faisant une large place à l'activité de recherche. Les personnels de formation eux-mêmes se voient confrontés à un nouveau statut dominant, celui d'enseignant-chercheur, qui exige, pour y accéder, un doctorat. (Bourdoncle, 2009, p. 19-20)

Or, en Suisse, les Hautes écoles pédagogiques (HEP) ne sont pas rattachées à une université (à quelques exceptions près). Elles sont le symbole de la tertiarisation, fondé sur la souveraineté cantonale en matière d'éducation et de formation, lorsque la formation est « modularisée et axée sur des standards et compétences professionnelles » et qu'elle s'accomplit «sur une base scientifique en se fondant sur des théories scientifiques et en transmettant des connaissances sur des méthodes scientifiques » (CDIP, 2008, p. 23-24).

Dans notre institution de formation, la HEP-BEJUNE, l'opposition entre tertiarisation et universitarisation semble se manifester du point de vue du type de structure et du statut des formateurs. Il y a cependant convergence entre ces deux notions, en ce qui concerne l'enjeu majeur de la professionnalisation du métier d'enseignant: la mise en relation réussie entre recherche et enseignement au sein de la formation.

Le rattachement de la formation professionnelle à l'enseignement supérieur relie symboliquement les professions au monde de la recherche, qui peut être plus ou moins élémentaire ou fondamentale, mais qui permet le développement des connaissances et des compétences et qui incline à la réflexion indépendante des considérations pratiques, commerciales et politiques. (Giannini et Gadea, 2006, p. 31)

Dans les faits, ce processus de tertiarisation des instituts suisses de formation des enseignants a été initié dès le début des années 1990 par la CDIP $(1993,1995)$. Celui-ci a entraîné la disparition des Écoles normales au profit d'institutions de niveau tertiaire, principalement des Hautes écoles pédagogiques (HEP), indépendantes des universités. À titre d'exemple, la HEP-BEJUNE s'est constituée par voie concordataire le 5 juin 2000, à partir de trois anciennes structures cantonales distinctes : l'École normale de Bienne (Berne), l'Institut pédagogique de Porrentruy (Jura), l'École normale du canton de Neuchâtel.

Ce processus de tertiarisation avait pour objectif principal d'améliorer la qualité de la formation en allongeant la durée des études, en élaborant de nouveaux programmes de formation de niveau universitaire, tout en rendant sa structure compatible avec le système de Bologne (calendrier académique, système d'accréditation ECTS [European Credits Transfer System], mastérisation de la formation des 
enseignants du second degré). La mobilité des étudiants durant leur formation y serait favorisée, mais aussi leur employabilité intercantonale, voire européenne.

Pour obtenir la reconnaissance des diplômes délivrés, la HEP-BEJUNE, comme ses institutions homologues de formation, a dû engager une procédure visant l'accréditation institutionnelle par la CDIP, concrétisée par le dépôt d'un dossier soumis pour évaluation. Face à un dossier aussi volumineux et diversifié dans la nature des documents, que beaucoup de nouveaux écrits sont venus densifier, comment organiser ces données de manière facilitante pour le chercheur?

\section{Cadre conceptuel : un outil au service de l'analyse discursive des textes institutionnels}

Pour l'analyse de notre corpus, nous avons écarté l'analyse de contenu au profit de l'analyse discursive pour éviter une posture illustrative, car l'analyse discursive est :

au cour des relations qui existent entre un comportement culturel et des discours sociaux. C'est dans ce cadre que le discours est conçu [...] comme un ensemble d'énoncés considérés dans leur dimension interactive, leur pouvoir d'action sur autrui, leur inscription dans une situation d'énonciation dont les paramètres sont : l'énonciateur, l'allocutaire, le moment de l'énonciation et le lieu de l'énonciation. (Barry, 2002, p. 4)

Le sens du discours n'est pas transparent à lui-même, mais construit par l'auteur en fonction de ses repères, de sa fonction et du contexte.

Plutôt qu'à une théorisation formelle, notre recherche s'apparente davantage à un essai (Desjardins et Hensler,2009) sur la manière de structurer la mise en dialogue des retraductions de la professionnalisation à différents niveaux textuels.

Pour préparer l'analyse du corpus, le dossier de reconnaissance de la HEP-BEJUNE et ses extensions, nous avons construit une modélisation des dispositifs de son système de formation et des textes qui en sont à l'origine, selon le modèle de Maxwell (1999). Nous l'avons choisi pour sa souplesse d'utilisation, l'absence de règles strictes et de contraintes. Il facilite une démarche empirique qui s'appuie sur des tâtonnements, à partir de conceptions a priori. Les rapports entre l'ensemble de ces textes et ces dispositifs rendaient visibles des axes d'analyse possibles pour soutenir des comparaisons intra et interinstitutionnelles et fournir des critères objectivables d'organisation et de tri du corpus des documents en fonction de trois dimensions : leur finalité; leur adressage et leur registre textuel. Prendre en considération ces trois dimensions permet de mettre au jour, dans l'analyse, d'éventuelles tensions ou rapports de force entre des documents de nature diverse ou des écarts entre le prescrit, les intentionnalités et le réel. Nous procèderons ci-dessous à la description de l'outil avant d'en illustrer une application possible.

\subsection{Description de l'outil d'analyse : les composantes et le fonctionnement}

Tout discours instituant une réforme de la formation imprègne les consciences et modèle les dispositifs et les identités pédagogiques. Pour Bernstein (2007), « des réformes éducatives différentes projettent des identités pédagogiques différentes. Toute réforme est vue comme une lutte entre groupes pour imposer 
une identité/ou des identités pédagogiques différentes» (p. 3). Pour Weinberg (2009), l'objectif de la rhétorique, qui confine à l'art pour certains, est de convaincre ou séduire par une argumentation pertinente, sans nécessité d'être scientifique. Elle s'appuie sur l'analogie et la métaphore, pour faire passer une idée.

Ainsi, la rhétorique de professionnalisation est aujourd'hui disséminée à travers les discours politiques à visée stratégique, les écrits institutionnels et les productions des étudiants pour dessiner les contours des dispositifs de formation :

[...] On se retrouve devant une conception ou un modèle de la professionnalisation de la formation encore en construction. Son élaboration semble se faire par étapes. Chaque étape récupère et intègre une des dimensions de la professionnalisation, dont il convient de tracer l'origine dans les travaux de recherche [...]. (Wentzel et Mellouki, 2010, p. 4)

Nous définirons la rhétorique de professionnalisation comme un ensemble de composantes lexicales qui traduisent les significations inhérentes au processus de professionnalisation ainsi que leurs effets potentiels. Elles sont déclinées dans la Figure 1.

Il faut toutefois être conscient que, dans l'analyse de ces discours, des tensions possibles sont à prendre en considération, entre injonctions à la professionnalisation et réalité du processus dans les dispositifs, entre les perceptions des acteurs et des étudiants. Cela a impliqué, pour nous, de construire un modèle qui donne à voir deux niveaux de tri et de lecture possibles du corpus dans une double vision, statique (états des lieux des structures, des écrits, des marqueurs de la rhétorique de professionnalisation) et dynamique (mise en rapport de ces composantes).

Notre outil d'analyse du dispositif de formation (voir Figure 1) se décline en trois composantes qui peuvent être prises pour elles-mêmes dans leur état statique ou mises en relation dynamique :

a) un dispositif de formation qui repose sur quatre domaines, celui des sciences de l'éducation (SED), celui de la recherche (RECHERCHE), celui de la formation sur le terrain (PRATIQUE) et celui des didactiques et des connaissances disciplinaires (DIDACTIQUE);

b) une typologie des écrits, à quatre niveaux, porteuse des discours de la rhétorique de professionnalisation. Nous développerons cette composante plus loin dans le texte en raison de sa fonction clé dans la recherche;

c) des marqueurs de la rhétorique de professionnalisation en rapport avec la problématique de la recherche spécifiée supra.

Le fonctionnement de cet outil se veut tout d'abord pragmatique. Il permet d'opérer des choix ciblés des composantes qui seront convoquées dans l'analyse : domaines du dispositif de formation; textes appartenant à un ou plusieurs des quatre niveaux d'écrits institutionnels; marqueurs de la rhétorique. Ces différentes composantes peuvent être analysées pour elles-mêmes.

Ensuite, lorsque l'analyse appelle leur croisement, cet outil permet de mieux identifier les mises en rapport possibles, intra et/ou intercomposantes, en fonction des objets de recherche construits par les chercheurs, de leurs objectifs et des cadres théoriques qu'ils convoquent. 


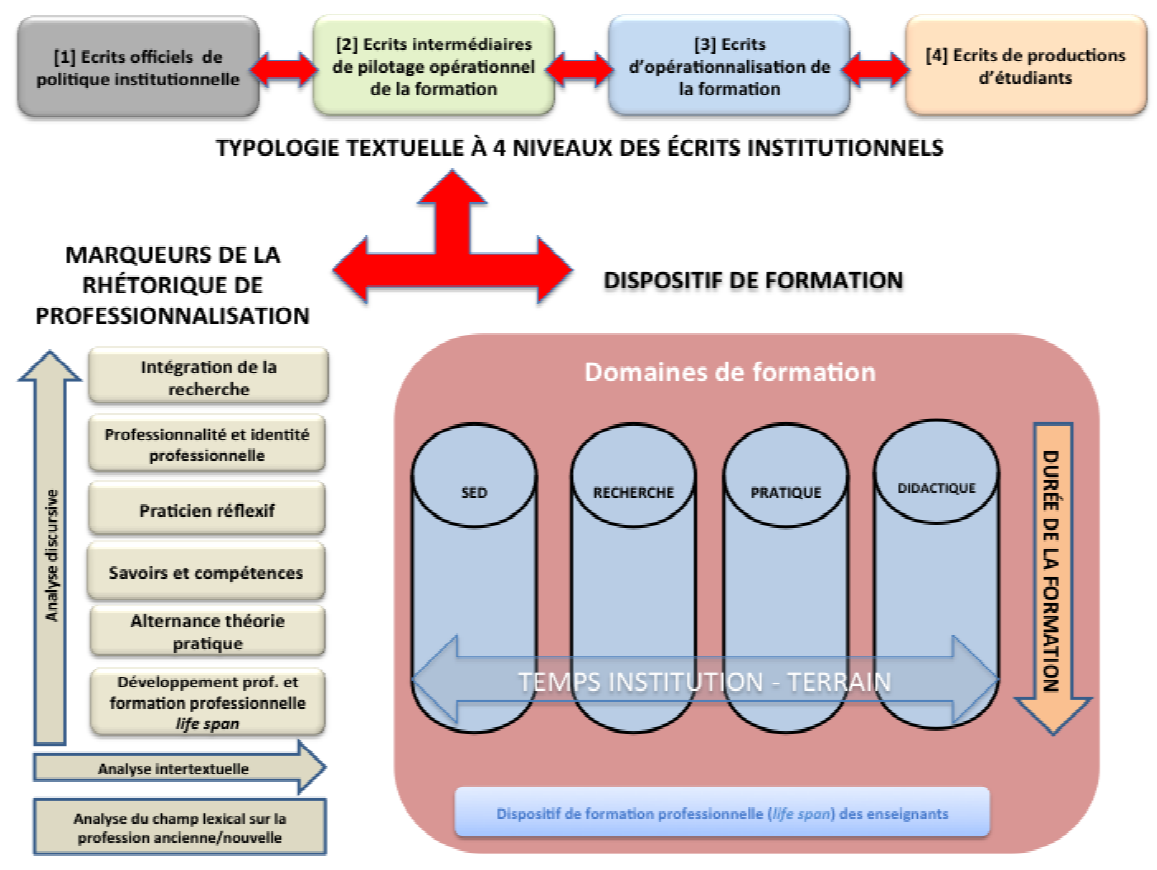

\section{Figure 1}

Outil au service de l'analyse du dispositif de formation de la HEP-BEJUNE.

Nous allons d'abord expliciter la construction de la typologie des écrits sur laquelle s'appuie notre modèle, puis nous allons montrer comment cet outil peut être utilisé à l'aide d'un exemple.

\subsection{Des étapes de construction d'une typologie des écrits à ses finalités}

a) Notre corpus initial de textes : les classeurs de reconnaissance CDIP de la HEP-BEJUNE

Dans ses Instructions pour l'élaboration d'une demande de reconnaissance de diplômes d'enseignement (CDIP, 2007) pour les degrés primaires et secondaires I et II, la CDIP exigeait que les institutions candidates fournissent dix fiches qui lui permettraient de fournir un rapport évaluatif sur la conformité des documents produits avec ses standards prescrits. Ces fiches portaient sur des Généralités relatives à la genèse et l'ancrage politique de l'institution, la Structure de la formation, les Objectifs et contenus de la formation, la Relation entre théorie et pratique, l'Enseignement et recherche, la Formation pratique, la Durée de la formation, les Conditions d'admission, la Réglementation liée à l'obtention du diplôme et à celle liée au personnel. Un exemplaire du classeur de reconnaissance BEJUNE, composé de plusieurs centaines de documents qui renseignent ces fiches, a constitué notre corpus de données de base. D’un point de vue méthodologique, comment organiser ce corpus?

b) De la nécessité de procéder à une typologie des écrits

Sur la base d'une adéquation avec les prescriptions établies (Faivre, 2012, p. 19), une procédure de confirmation de reconnaissance doit être engagée par les HEP tous les sept ans à l'intention de la CDIP. 
Les formations primaire et secondaire BEJUNE ont obtenu la reconnaissance CDIP, respectivement en 2005 et en 2012.

Peut-on toutefois se contenter de penser qu'une institution réponde aux critères de professionnalisation par le seul fait qu'elle soit accréditée en tant que telle par la CDIP? Sous l'angle de l'analyse quantitative comparée des structures, des programmes et des contenus de formation de deux institutions de formation à l'enseignement, on peut répondre par l'affirmative, car l'intention de toute institution de cet ordre est de donner à voir qu'elle est conforme aux attentes de l'entité politico-éducative qui la régit.

Or, d'un point de vue qualitatif, on ne peut prétendre saisir les enjeux de la professionnalisation sans prendre en considération les conditions, les circonstances, les facteurs et les acteurs qui ont contribué à produire les documents du corpus de données. Pour Cossette (2004), « les acteurs de l'organisation sont au cœur du processus de [sa] structuration. Leurs actions sont influencées par leur connaissance profondément subjective des contextes externe et interne de l'organisation, qu'ils ont eux-mêmes contribué à construire ou enacter» (p.4). De ce fait, chaque élément du corpus doit être interprété non seulement en fonction de son contenu, mais également en fonction de son adressage : adressé par (le produit, dans un contexte donné, de la pensée d'une personne, d'un groupe de formateurs, d'un responsable de formation, d'une institution politico-éducative, etc.), adressé à (étudiants, formateurs, responsables de formation, instances de direction, formateurs en établissement, sphère publique, etc.) et adressé pour quoi (finalité des écrits).

En effet, selon les théories en analyse du discours (Barry, 2002), un énoncé se présente comme un texte : un mode d'organisation spécifique « qu'il faut étudier comme tel en le rapportant aux conditions dans lesquelles il est produit. Considérer la structure d'un texte en le rapportant à ses conditions de productions, c'est l'envisager comme discours » (Grawitz, 1990, cité dans Barry, 2002, p. 1), sachant qu'un discours est une construction qui se caractérise par des acteurs, des objets, des propriétés, des événements sur lesquels il s’opère (Maingueneau, 1989, cité dans Barry, 2002).

Pour intégrer ces trois paramètres dans un répertoire interprétatif au service de l'analyse intertextuelle, la construction d'une typologie des écrits, constitutifs de notre corpus, s'est imposée.

c) Les catégories constitutives de typologie des écrits

Dans un premier temps, nous avons utilisé une approche inductive (Paillé et Mucchielli, 2008) pour construire les catégories de notre typologie, à la suite d'une lecture flottante du corpus. Quatre catégories ont alors émergé (voir Figure 1) :

1. les écrits officiels de politique institutionnelle;

2. les écrits intermédiaires de pilotage opérationnel de la formation;

3. les écrits d'opérationnalisation de la formation;

4. les écrits de production d'étudiants.

Dans un second temps, nous avons mis ces catégories à l'épreuve des trois filtres: l'auteur/les auteurs, l'adressage et la finalité des écrits. Concrètement, nous avons ventilé nos textes en fonction de la présence de l'un ou l'autre de ces filtres. Dans cette logique, les documents qui ne répondaient à aucun des trois filtres ont été exclus de l'analyse. 
Passer ces textes au crible des filtres a permis de vérifier la pertinence des catégories construites $a$ priori, ils ont également contribué à en faire émerger une nouvelle. Elle correspond à l'ensemble des écrits officiels de politique éducative, externes à l'institution, qu'ils s'ancrent dans un contexte cantonal, national ou international. Ils sont prescriptifs ou de recommandation et chapeautent les différents écrits produits par l'institution elle-même. Le tableau ci-dessous (voir Tableau 1) rend compte de la typologie et renseigne précisément les trois filtres.

\section{Tableau 1}

Typologie des écrits.

\begin{tabular}{|c|c|c|c|c|c|}
\hline \multicolumn{6}{|c|}{ Catégories constitutives de la typologie } \\
\hline Filtres & $\begin{array}{l}\text { Écrits officiels de } \\
\text { politique éducative }\end{array}$ & $\begin{array}{l}\text { Écrits officiels } \\
\text { de politique } \\
\text { institutionnelle }\end{array}$ & $\begin{array}{l}\text { Écrits intermédiaires } \\
\text { de pilotage } \\
\text { opérationnel de la } \\
\text { formation }\end{array}$ & $\begin{array}{l}\text { Écrits d'opération- } \\
\text { nalisation de la } \\
\text { formation }\end{array}$ & $\begin{array}{l}\text { Écrits de production } \\
\text { d'étudiants }\end{array}$ \\
\hline Auteur(s) & $\begin{array}{l}\text { Gouvernement, } \\
\text { services cantonaux, } \\
\text { institutions } \\
\text { internationales }\end{array}$ & $\begin{array}{l}\text { Rectorat } \\
\text { et/ou comité } \\
\text { stratégique, } \\
\text { exécutif cantonal }\end{array}$ & $\begin{array}{l}\text { Responsables de } \\
\text { la formation ou de } \\
\text { I'organisation de la } \\
\text { formation }\end{array}$ & $\begin{array}{l}\text { Formateurs } \\
\text { (individuellement } \\
\text { ou collectivement) }\end{array}$ & $\begin{array}{l}\text { Étudiants } \\
\text { (individuellement } \\
\text { ou collectivement) }\end{array}$ \\
\hline Adressage & $\begin{array}{l}\text { Instituts de } \\
\text { formation des } \\
\text { enseignants }\end{array}$ & $\begin{array}{l}\text { Personnel } \\
\text { institutionnel, } \\
\text { étudiants, public }\end{array}$ & $\begin{array}{l}\text { Formateurs, } \\
\text { étudiants, } \\
\text { formateurs en } \\
\text { établissement, } \\
\text { public }\end{array}$ & Étudiants, pairs & $\begin{array}{l}\text { Formateurs, } \\
\text { formateurs en } \\
\text { établissement, } \\
\text { institution, soi, pairs }\end{array}$ \\
\hline Finalités & $\begin{array}{l}\text { Cadrage } \\
\text { (prescriptif ou de } \\
\text { recommandation) } \\
\text { de l'institution }\end{array}$ & $\begin{array}{l}\text { Visions } \\
\text { stratégiques } \\
\text { et politiques, } \\
\text { pilotage } \\
\text { stratégique et } \\
\text { opérationnel }\end{array}$ & $\begin{array}{l}\text { Pilotage } \\
\text { opérationnel de la } \\
\text { formation, faciliter } \\
\text { l'accès à l'institution }\end{array}$ & $\begin{array}{l}\text { Déclinaison } \\
\text { opérationnelle } \\
\text { des réquisits } \\
\text { institutionnels } \\
\text { et moyens mis } \\
\text { en œuvre pour } \\
\text { répondre à la } \\
\text { demande }\end{array}$ & $\begin{array}{l}\text { Légitimer un } \\
\text { processus de } \\
\text { formation }\end{array}$ \\
\hline
\end{tabular}

L'ensemble des documents produits par les différentes institutions partenaires de la recherche FNS de Wentzel (2012) peut être organisé selon cette typologie pour pouvoir les faire ensuite dialoguer entre eux et de mettre au jour d'éventuelles tensions, contradictions entre les catégories. Dès lors que l'analyse est organisée et contextualisée, des comparaisons de textes interinstitutionnels peuvent être envisagées.

\section{L'application de l'outil dans le cheminement méthodologique d'une recherche}

L'outil s'est révélé comme une aide précieuse dans la sélection et la mise en relation des textes relatifs à la place de la recherche en formation, en tant que dimension incontournable de la professionnalisation de la formation des enseignants. Comment est-elle énoncée et retraduite dans les discours institutionnels 
de niveaux différents, produits dans les contextes socioculturels et linguistiques propres au système éducatif helvétique? Il en découle l'existence d'une grande diversité des filières de formation des enseignants. La description des structures internes de ces filières montre qu'il existe des conceptions polysémiques, des significations qui ne sont pas toujours partagées (Lehmann, 2006) et une répartition différenciée des contenus de formation. Ainsi, l'interprétation des pourcentages attribués aux différents domaines de formation dans les Hautes écoles pédagogiques de Suisse romande (HEP), les Pädagogischen Hochschulen de Suisse alémanique (PH) et les Instituts universitaires de formation des enseignants (IUFE) est fortement liée à des terminologies et à des conceptions différenciées selon les régions linguistiques du pays et, même au-delà, plus localement, selon le canton dans lequel on se situe.

La comparaison formelle, sur la base d'indicateurs visibles, ne renseigne donc pas sur la véritable place de la recherche en formation, sur sa fonction, sur sa nature, sur la force de ses frontières avec d'autres domaines ou sur son degré de classification (Bernstein, 2007), ni sur son degré d'intégration à la formation en général. Selon cette logique de mise en dialogue, il devient inapproprié de comparer le volume attribué à chaque domaine de formation en seuls termes de crédits ECTS ou de volumes d'heures de formation, car il n'existe pas de correspondance absolue dans les significations données à ces domaines selon les différences de cultures institutionnelles (Lehmann, Criblez, Guldimann, Fuchs et Périsset Bagnoud, 2007). Par exemple, l'interprétation de la notion d'ECTS apparait dans une acception différenciée en ce qui concerne le volume de travail qu'elle représente pour l'étudiant :

- à la HEP-BEJUNE, le temps de travail est formellement partagé entre temps de cours (séminaires ou ateliers) et temps de travail autonome selon la règle suivante : $1 \mathrm{ECTS}=12$ périodes de cours de 45 minutes ( 9 heures) et entre 16 et 21 heures de travail autonome;

- à l'IUFE, la valeur d'un crédit est définie selon les recommandations de la CRUS ${ }^{3}$ ainsi un crédit équivaut à une charge de travail de 25 à 30 heures laissant plus de marge de manœuvre dans les modalités d'enseignement des différentes unités de formation.

On peut relever également, au-delà des différences interinstitutionnelles, des variations à l'interne des institutions, la valeur d'un crédit SED ou RECHERCHE étant différente de celle d'un crédit de formation pratique.

Les premiers constats montrent que la recherche est formellement identifiable en tant qu'entité spécifique de formation, mais que son volume en termes de crédits ne suffit pas à évaluer son intégration dans tous les domaines de formation.

Ensuite, nous avons centré notre attention sur les différentes conceptions officielles au sujet de la recherche en tant qu'instrument obligatoirement intégré dans une formation professionnalisante.

À l'aide de l'outil, nous avons pris la mesure d'une réalité double : une réalité visible (l'énoncé explicite, dans des textes de différents niveaux, d'un nombre d'ECTS attribué à un domaine par exemple) et une réalité invisible (les indicateurs permettant de définir une présence implicite de la recherche dans d'autres domaines de formation que celui de la recherche lui-même). Cette double réalité ne renseigne pas sur la véritable place de la recherche dans la formation.

Méthodologiquement, notre outil nous a permis un recodage en termes d'éléments constitutifs du domaine de la recherche et sa mise en relation avec les différents niveaux textuels des documents 
institutionnels. Les données gagnent ainsi en pertinence et en lisibilité pour les chercheurs. Notre outil nous a amenés à une hypothèse de travail se rapportant à une conception spécifique de la recherche, en tant que composante du processus de professionnalisation, sous-jacente aux modes de retraduction des discours de différents niveaux. Il nous a amené à une nouvelle sélection de textes à mettre en tension dans une analyse de la rhétorique de professionnalisation à l'aide d'une cadre théorique issu de la linguistique et de la psychologie sociale, nous permettant d'analyser les contradictions conceptuelles qu'ils contiennent. Les contradictions sont inhérentes à la pensée humaine et sont une clé de l'élaboration des systèmes de formations. En cela, elles sont révélatrices de la pensée des décideurs et des dirigeants d'institutions de formation (Studer, 2012). Toujours selon cet auteur, «les concepts imprègnent le discours et le discours imprègne les concepts : il semble évident qu'il existe une forte interaction entre les deux phénomènes analytiques" (Studer, 2012, p. 124). Les contradictions conduisent ainsi à des luttes entre acteurs et amènent des stratégies de résolution qui dessinent les cadres institutionnels de la professionnalisation. Nous nous sommes concentrés sur l'analyse des contradictions dans les discours de niveau 1 et 2 de notre outil, mais obligés parfois à explorer un troisième niveau. En effet, des textes aux finalités semblables ne se situaient pas obligatoirement au même niveau dans notre typologie textuelle. Nous avons dû ainsi porter notre attention tant sur la finalité des documents que sur leur contenu, leurs auteurs et leurs destinataires pour les aborder dans une perspective comparative.

Nos observations montrent que l'on retrouve globalement les mêmes contradictions à chaque niveau textuel. Elles montrent aussi que la formation à la recherche est l'objet d'une rhétorique inclusive mais qu'en réalité, elle est reléguée au rang d'outsider ou de satellite en regard de la formation pratique dans les dispositifs de formation.

\section{Conclusion et perspectives}

Notre contribution visait à présenter et à soumettre au débat un outil méthodologique élaboré empiriquement, utile à l'organisation d'un corpus de textes soumis à l'analyse et à leur mise en rapport dans une analyse intertextuelle, avec des composantes structurelles et conceptuelles de plusieurs dispositifs de formation et avec des marqueurs de la professionnalisation qui font consensus. À ce stade, nous ne sommes pas encore à même de démontrer sa généralisation à d'autres recherches. Néanmoins, nous pouvons en tirer quelques conclusions.

Cet outil a fonctionné comme ressource à l'ensemble du groupe de chercheurs dans la détermination de leurs objets de recherche et comme garant de la cohérence d'ensemble des différentes recherches conduites.

Les premiers résultats, issus de l'exemplification, viennent nuancer l'absolu des propos écrits, par la mise en dialogue des différents niveaux textuels, les écarts entre le prescrit des textes et leur retraduction dans l'opérationnalisation de la formation.

Il a permis d'aborder la complexité de la sélection de discours institutionnels significatifs par une vision globale constante des composantes de la recherche - en rapport avec ses objectifs - pour explorer des mises en rapport possibles et pour amener des éléments de compréhension à l'apparente évidence de certaines comparaisons quantitatives. Enfin, il nous a permis de focaliser nos recherches sur des zones d'ombre peu analysées de la rhétorique de la professionnalisation. 
Nous avons conscience que l'analyse de la rhétorique de professionnalisation ne peut reposer seulement sur des analyses textuelles et intertextuelles. Elle doit être approfondie par des entretiens avec les acteurs de chaque niveau textuel (auteurs ou lecteurs) pour mettre au jour leurs modes de retraduction des discours de niveau supérieur, constitutifs de leurs conceptions de la professionnalisation. C'est là une piste de développement de notre outil.

Nous faisons toutefois l'hypothèse que cet outil est généralisable à d'autres institutions de formation des enseignants, puisqu'il permet d'organiser les textes institutionnels pour les préparer à l'analyse.

\section{Références}

Barry, A. O. (2002). Les bases théoriques en analyse du discours. Repéré à https://depot.erudit.org/id/002331dd

Bernstein, B. (2007). Pédagogie, contrôle symbolique et identité : théorie, recherche, critique (traduit par G. Ramognino-Le Déroff et P. Vitale). Québec, QC : Presses de l'Université Laval.

Blandin, B. (2002). La construction du social par les objets. Paris : Presses universitaires de France.

Bourdoncle, R. (2009). L'universitarisation. Structures, programmes et acteurs. Dans R. Étienne, M. Altet, C. Lessard, L. Paquay et P. Perrenoud (dir.), L'université peut-elle vraiment former les enseignants? Quelles tensions? Quelles modalités? Quelles conditions? (p. 19-28). Bruxelles : De Boeck.

Conférence suisse des directeurs cantonaux de l'instruction publique (CDIP). (1995). Recommandations relatives à la formation des enseignant(e)s et aux hautes écoles pédagogiques. Repéré à http://edudoc.ch/record/25493/files/19951026f.pdf

Conférence suisse des directeurs cantonaux de l'instruction publique (CDIP). (2007). Instructions pour l'élaboration d'une demande de reconnaissance de diplômes d'enseignement pour les écoles de maturité. Berne : CDIP.

Conférence suisse des directeurs cantonaux de l'instruction publique (CDIP). (2008). Profession enseignante : analyse des changements et conclusions pour l'avenir. Repéré à http://edudoc.ch/record/27310/files/StuB27B.pdf

Cossette, P. (2004). L'organisation : une perspective cognitiviste. Québec, QC : Presses de l'Université Laval.

Cossette, P. (2008). La cartographie cognitive vue d'une perspective subjectiviste : mise à l'épreuve d'une nouvelle approche. AIMS M@n@gement, 11(3), 259-281.http://dx.doi.org/10.3917/mana.113.0259

Desjardins, J. et Hensler, H. (2009). À la recherche d'une cohérence dans les programmes de formation à l'enseignement : le rôle des acteurs et la prise en compte des caractéristiques organisationnelles. Dans R. Étienne, M. Altet, C. Lessard, L. Paquay et P. Perrenoud (dir.), L'université peut-elle vraiment former les enseignants? Quelles tensions? Quelles modalités? Quelles conditions? (p. 145-159). Bruxelles : De Boeck.

Faivre,J.-P. (2012). La Haute école pédagogique BEJUNE : une entité tricantonale. Enjeux pédagogiques, (19), 5-10. Repéré à http://www.hep-bejune.ch/boutique/enjeux-pedagogiques/pdf/HEPB BULL No19.pdf

Giannini, M. et Gadea, C. (2006). Introduction. Savoir, travail E̋ société, 4(2), 7-42.

Lehmann, L. (2006). Harmonisation des habilitations à enseigner dans le domaine des disciplines et des degrés d'enseignement : expertise effectuée sur mandat de la commission Formation de la Conférence suisse des recteurs des hautes écoles pédagogiques. Repéré à https://www.swissuniversities.ch/fileadmin/swissuniversities/Dokumente/FR/PH/Dokumente/2006 Bericht Harmonisierung fr.pdf

Lehmann, L., Criblez, L., Guldimann, T., Fuchs, W. et Périsset Bagnoud, D. (2007). Les formations à l'enseignement en Suisse. Rapport dans le cadre du monitorage de l'éducation 2006. Aarau : Centre suisse de coordination pour la recherche en éducation.

Maingueneau, D. (1989). Genèse du discours (2éd.). Bruxelles : Mardaga.

Maxwell, J.-A. (1999). La modélisation de la recherche qualitative. Une approche interactive. Fribourg: Presses universitaires de Fribourg. 
Paillé, P. et Mucchielli, A. (2008). L'analyse qualitative en sciences humaines et sociales ( ${ }^{\mathrm{e}}$ éd.). Paris : Armand Colin.

Studer, P. (2012). Conceptual contradiction and discourses on multilingualism. Dans P. Studer et Y. Werlen (dir.). Linguistic diversity in Europe. Current trends and discourses (p. 115-136). Berlin : Walter de Gruyter.

Tardif, M. et Borges, C. (2009). L'internalisation de la professionnalisation de la formation à l'enseignement secondaire et ses retraductions dans des formes sociales nationales. Dans R. Hofstetter et B. Schneuwly (dir.), Savoirs en (trans)formation. Au cour des professions de l'enseignement et de la formation (p. 109-139). Bruxelles : De Boeck. http://dx.doi.org/10.3917/dbu.hofst.2009.01.0109

Tilman, F. (2005). L'analyse institutionnelle. Repéré à http://www.legrainasbl.org/index.php?option=com content\&view=art icle\&id=118:1analyse-institutionnelle-i-fondements\&catid=9\&Itemid=103

Weinberg, A. (2009). Aux sources de l'éloquence. Sciences humaines, (209), 31-35.

Wentzel, B. (2012). La professionnalisation de la formation des enseignants : le cas de la Suisse [№ 136849]. Projet du Fonds national suisse de la recherche scientifique (FNS) 2012-2014. FNS, Berne.

Wentzel, B. et Mellouki, M. (2010). Internationalisation de la professionnalisation de la formation des enseignants et retraductions nationales : le cas de la Suisse. Actes du congrès de l'Actualité de la recherche en éducation et en formation (AREF), Genève. Repéré à https://plone.unige.ch/aref2010/symposiums-longs/coordinateurs-en-w/ professionnalisation-de-la-formation-des-enseignants-des-fondements-aux-retraductions-nationales/Internationalis ation\%20de\%201a\%20professionnalisation.pdf

Wittorski, R. (2014). Professionnalisation. Dans A. Jorro (dir.), Dictionnaire des concepts de la professionnalisation (p. 229231). Louvain-la-Neuve : De Boeck.

\section{Notes}

1 Contrairement à la France, il n'existe pas de ministère national de l'Éducation en Suisse. La Conférence suisse des directeurs cantonaux de l'instruction publique (CDIP) rassemble les ministres de l'Éducation des 26 cantons helvétiques. Elle a pour mission de coordonner et de dynamiser le système scolaire suisse, très décentralisé, du fait de la souveraineté cantonale en matière d'éducation.

2 La Haute école pédagogique des cantons de Berne, Jura et Neuchâtel (HEP-BEJUNE), Suisse.

3 Conférence des directeurs des universités suisses (CRUS).

\section{Pour citer cet article}

Stumpf, A., Joliat, F. et Perrin, D. (2016). Comment analyser les traces de la professionnalisation dans les écrits des institutions de formation à l'enseignement? Formation et profession, 24(2) 33-44.

http://dx.doi.org/10.18162/fp.2016.343 